\title{
Vibrational properties of boron nitride nanotubes: Effects of finite length and bundling
}

\author{
Ludger Wirtz and Angel Rubio \\ Donostia International Physics Center and Department of Material Physics, University of the \\ Basque Country, Po. Manuel de Lardizabal 4, 20018 Donostia - San Sebastián, Spain
}

\begin{abstract}
We present $a b$ initio calculations of phonons in single-wall boron nitride nanotubes. Raman and infrared active modes of isolated and infinitely long tubes are evaluated according to the non-symmorphic rod groups of BN nanotubes. For tubes of finite length, the selection rules are less restrictive and give rise to additional modes which may be observed in Raman and IR spectroscopy with an intensity depending on the tube length. Bundling of tubes is shown to have little effect on the phonon frequencies. However, arranging tubes in a large periodic array (larger than the wave-length of incoming light) gives rise to a strong frequency shift (LO-TO splitting) of some modes due to the establishing of a macroscopic electric field. Modes of $A_{1}$ symmetry experience a shift for laser light along the tube axis and $E_{1}$ modes are split for light incidence in the perpendicular direction.
\end{abstract}

Keywords: BN-nanotubes, Raman spectroscopy, infrared spectroscopy, phonons, LO-TO splitting

\section{INTRODUCTION}

Boron nitride is isoelectronic to carbon and displays - among others - a graphite-like hexagonal phase (h-BN) which has been extensively studied by various spectroscopic methods..$^{1-6}$ The elastic constants are very similar to carbon (although smaller), but the polar nature of the BN-bond leads to significant changes in the electronic structure of h-BN as compared to graphite. While graphite is a semimetal (zero band gap in the single graphenesheet), h-BN has a large band gap of about $5.5 \mathrm{eV} .{ }^{3}$ Furthermore, its high thermal stability and relative chemical inertness, distinguishes it from its carbon counterpart.

The structure of a carbon nanotube can be explained by rolling up a single graphene sheet. It is natural to do the same with a single hexagonal BN-sheet and construct a BN-nanotube which is isoelectronic to Cnanotubes but should carry over some of the characteristic differences of h-BN with respect to graphite. Indeed, tight-binding ${ }^{7}$ and first-principles calculations ${ }^{8}$ demonstrated that BN-tubes are stable and have a band gap similar to h-BN, independent of the tube diameter and chirality. Furthermore, the band gap is independent of the number of walls in multi-wall nanotubes as long as the inner diameter is above $0.8 \mathrm{~nm}$.

First synthesis of multi-wall BN-tubes was reported in 1995. ${ }^{11}$ By now, BN-tubes are routinely produced in several groups. ${ }^{12-18}$ The production of single-wall BN-tubes in gram quantities ${ }^{19}$ is a further mile-stone toward applications of BN-nanotubes. On this way, BN-nanotubes face the same problems as its carbon brethren: The raw product at the end of the production process contains tubes of various lengths, diameters and chiralities some of which are isolated while others form bundles. This tube material may furthermore be contaminated by catalyst particles and raw material from the beginning of the production process. Further processing at this stage requires detailed information from spectroscopy.

Several spectroscopic methods are commonly used for the identification and characterization of nanotube samples: High resolution transmission electron spectroscopy (HRTEM) allows for a quick view at the scene with almost atomic resolution. Scanning tunneling microscopy/spectroscopy (STM/STS) allow to get atomic resolution and to map the electronic structure to the underlying nanotube geometry. ${ }^{20}$ In optical spectroscopy, using laser light, the spacial resolution is lost. However, alternative information about the band-structure and the vibrational properties of the constituents can be gained. Optical absorption spectroscopy probes the electronic band structure by direct excitation of an electron from the valence to the conduction band. Since BN-tubes

Email: lwirtz@sc.ehu.es 
have a wide band-gap, either multiphoton processes or UV light are necessary for this process to occur in BNnanotubes. Recent spectrofluorimetric data on SWNT suspended in an aqueous surfactant combined with Raman and optical absorption has been able to provide information about the tube chirality. ${ }^{21}$ At lower energy, infrared (IR) absorption spectroscopy probes the direct excitation of phonons. Finally, Raman spectroscopy probes the excitation of phonons by measuring the frequency shift in inelastically scattered laser light.

We investigate the application of the latter two spectroscopic methods (Raman and IR) for the characterization of BN-nanotubes. For carbon materials, Raman scattering is resonantly enhanced because the band gap is zero or very small and electrons can be excited from the valence to the conduction band. This results in a high intensity of the spectra and a sensitive dependence on the laser frequency. Therefore, Raman spectroscopy has become an accurate, highly diameter selective tool for the characterization of carbon nanotubes. ${ }^{22,23}$ In BN-tubes, Raman scattering is non-resonant due to the large band gap of the tubes. The resulting spectra are therefore weaker in intensity and must be carefully separated from a possible overlap by resonant Raman scattering from contaminants. ${ }^{24}$ On the other hand, in BN-tubes, the efficiency of IR-absorption spectroscopy is enhanced by the polarity of the material and gives rise to much richer IR spectra than in the case of carbon tubes where the IR-spectra have very little structure and can hardly be distinguished from the IR-spectrum of graphite. $^{25}$

For the interpretation of measured spectra, ${ }^{24}$ an accurate knowledge of the phonon frequencies as a function of tube diameter and chirality is indispensable. In a recent paper, ${ }^{26}$ we have presented the first $a b$ initio calculations of phonons in ideal, isolated, infinitely long BN-nanotubes. In this paper, the emphasis lies on the effects of deviation from this ideal case, i.e., finite tube size and occurrence of tubes in bundles. Experimentally produced tubes have a finite length (typically between 200 and $400 \mathrm{~nm}^{24}$ ) which is shorter than the wave-length of IR light. In this case, the tubes appear as very large molecules rather than infinitely extended. This lowers the non-symmorphic rod group symmetry of the tubes ${ }^{27,28}$ to the point-group symmetry of a finite-size tube. Lower symmetry leads to a relaxation of selection-rules and gives rise to additional "allowed" Raman and IR active modes. The effect of bundling of tubes is difficult to treat exactly, because a super-cell for a bundle of, e.g., 10 tubes would be prohibitively large. As an approximation to the bundling effect, we study therefore the phonon frequencies in an (infinitely extended) close packed array of tubes. In this case, the polarity of BN allows for the coupling of certain modes to a self-induced macroscopic electric field (Lydanne-Sachs-Teller splitting ${ }^{29}$ ) which leads to a shift of some frequencies. This effect depends on the direction of the incoming laser light.

The structure of the paper is as follows: In section 2 we give some details on the calculation of phonon frequencies by density-functional perturbation theory and compare with other, more empirical methods. The results for ideal, infinitely long tubes are summarized in section 3 where also an intuitive explanation of the symmetry of Raman and IR active modes is given. In section 4 we present the frequencies of Raman and IR active phonon modes in tubes of finite length. In section 5 we discuss the expected Raman and IR spectra in a periodic array of densely packed tubes.
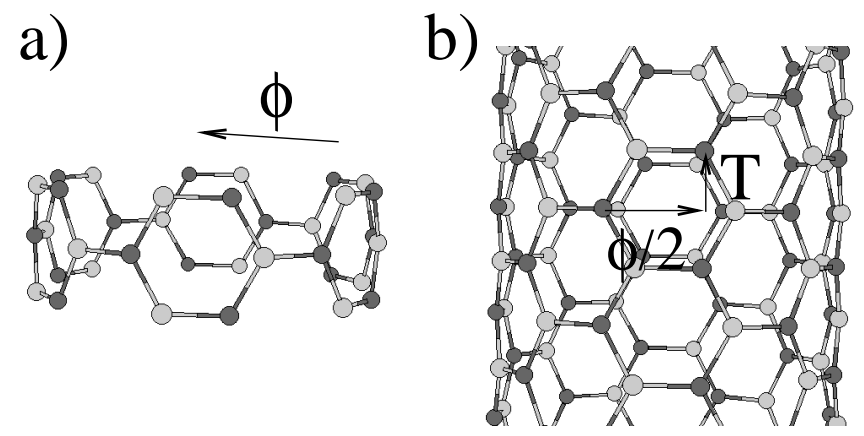

Figure 1. Comparison of the point-group symmetry of the unit cell with the space-group symmetry of the armchair BN-tubes. 


\section{METHOD OF CALCULATION}

The phonon frequencies $\omega$ as a function of the phonon wave-vector $q$ are the solution of the secular equation

$$
\operatorname{det}\left|\frac{1}{\sqrt{M_{s} M_{t}}} C_{s t}^{\alpha \beta}(q)-\omega^{2}(q)\right|=0 .
$$

$M_{s}$ and $M_{t}$ denote the atomic masses of atoms $s$ and $t$ and the dynamical matrix is defined as

$$
C_{s t}^{\alpha \beta}(q)=\frac{\partial^{2} E}{\partial u_{s}^{* \alpha}(q) \partial u_{t}^{\beta}(q)},
$$

where $u_{s}^{\alpha}$ denotes the displacement of atom $s$ in direction $\alpha$. The second derivative of the energy in Eq. (2) corresponds to the change of the force acting on atom $t$ in direction $\beta$ with respect to a displacement of atom $s$ in direction $\alpha$ :

$$
C_{s t}^{\alpha \beta}(q)=\frac{\partial}{\partial u_{s}^{* \alpha}(q)} F_{t}^{\beta}(q) .
$$

Note the dependence on $q$ of the dynamical matrix and the displacements. In an explicit calculation of the dynamical matrix by displacing each of the atoms of the unit cell into all three directions, a periodic supercell has to be used which is commensurate with the phonon wave length $2 \pi / q$. Fourier transform of the q-dependent dynamical matrix leads to the real space force constant matrix $C_{s t}^{\alpha \beta}(R)$. A phonon calculation starts with a determination of the dynamical matrix in real space or reciprocal space (Eq. (2)).

Before discussing how the dynamical matrix is constructed for BN nanotubes, it is instructive to look at how phonons have been calculated in carbon tubes. The easiest, very intuitive and computationally fast approach is the use of (real space) force constants that are fitted to experimentally measured phonon dispersion relations. A set of force constants up to 4th-nearest neighbor interaction was originally used to describe the phonon dispersion relation of graphite and has become the standard also for nanotubes. ${ }^{9,10}$ However, the agreement between experiment and theory is limited to certain regions in the first Brillouin zone. Furthermore, recent $a b$ initio calculations ${ }^{30}$ which agree almost perfectly with the experimental dispersion relation of graphite, have explicitly listed force constants and demonstrated that the cutoff with the 4th-nearest neighbor is questionable. A more precise, but also more costly, alternative to the use of a force constant approach is the explicit calculation of the dynamical matrix by tight-binding (see, e.g., ${ }^{31}$ ) or ab initio methods. ${ }^{30,32}$ For BN-tubes, phonons have been calculated $a b$ initio, ${ }^{24}$ by tight-binding, ${ }^{33}$ and by a valence shell model. ${ }^{34}$

We have chosen the ab initio approach for our calculation, because the tight-binding calculations for phonons in $\mathrm{C}$ nanotubes produced deviations of up to $100 \mathrm{~cm}^{-1}$ for the phonons in the high-frequency regime. We use density-functional theory (DFT) in the local density approximation (LDA) as implemented in the code ABINIT. ${ }^{35}$ Only valence electrons are treated explicitly. Core electrons are described by Troullier-Martins pseudopotentials. ${ }^{36}$ The wave-functions are expanded in plane waves with an energy cutoff at 80 Ry. Since the code requires the use of a three-dimensional supercell, the (infinitely long) tubes are ordered in a triangular periodic array. In order to simulate an isolated tube, we choose a large inter-tube distance of 14 a.u. For the simulation of the "solid of tubes", a distance of $6.5 \mathrm{a} . \mathrm{u}$ which corresponds roughly to the inter-sheet distance in $\mathrm{h}-\mathrm{BN}$ is chosen. The tube geometry is optimized (until the forces acting on all atoms are smaller than $5 \times 10^{-5}$ a.u.) and the stress along the tube axis is minimized by adjusting the super-cell size along the tube-axis.

The second derivatives in the dynamical matrix (Eq. (2)) are calculated in density-functional perturbation theory $(\mathrm{DFPT})^{37}$ as implemented in ABINIT. ${ }^{38}$ This means that the atomic displacements are not performed explicitly but treated as a perturbation. The equations for the first order change in the electron density can be cast into a form similar to the Kohn-Sham equations ${ }^{39}$ and must be solved self-consistently (i.e. adjusting the Hartree and the exchange-correlation potential with the change in density). In the case of (quasi) isolated tubes, only the displacement perturbation of one boron and one nitrogen atom needs to be calculated explicitly. The residual elements of the dynamical matrix are obtained by symmetry transformation employing the nonsymmorphic rod group symmetry of the tubes. In the case of the close-packed tubes, the exact symmetry is lost and all atomic displacement have to be calculated. 
Since BN is a polar material where $\mathrm{N}$ is slightly negatively charged and B slightly positive, the long-range character of the Coulomb potential gives rise to a macroscopic electric field $E$ for longitudinal optical phonons in the limit $q \rightarrow 0$. The dynamical matrix (Eq. 2) must therefore be corrected under inclusion of the Born effective charge tensor $Z_{s}^{* \alpha \beta}$ of the ions and the static dielectric tensor $\epsilon_{\infty}^{\alpha \beta}$. Both quantities can be calculated in $\mathrm{DFPT}^{37,38}$ from the macroscopic electric polarization $P$ of the medium through

$$
e Z_{s}^{* \alpha \beta}=\left.\Omega \frac{\partial P_{\alpha}}{\partial u_{s}^{\beta}(q=0)}\right|_{E=0}
$$

where $\Omega$ denotes the unit cell volume and

$$
\epsilon_{\infty}^{\alpha \beta}=\delta_{\alpha \beta}+\left.4 \pi \frac{\partial P_{\alpha}}{\partial E_{\beta}}\right|_{u_{s}(0)=0} .
$$

For (quasi) one dimensional systems like isolated tubes, no classical macroscopic electric can be induced through longitudinal optical phonons and the above mentioned corrections can be neglected. However, in section 5 it will be shown that the effect of electric-field corrections is very strong for some modes in large periodic arrays of tubes.

\section{RAMAN AND IR ACTIVE MODES IN INFINITELY LONG TUBES}

Since the photons of IR or visible light carry a momentum which is negligible compared to the momentum associated with atomic motion, only phonons with a wave-vector $q \approx 0$ (the " $\Gamma$-point" in reciprocal space) can be excited in first order Raman and IR absorption processes. Selection rules impose a further restriction on the number of IR and Raman active modes. Only modes that transform under symmetry operations as a quadratic form are Raman active and only modes that transform as a vector are IR active. Higher symmetry leads to stricter selection rules and thereby to a lower number of active modes. Raman and IR active modes of carbon nanotubes have been frequently evaluated based on the point group symmetry of the unit-cell. ${ }^{9}$ However, Alon pointed out ${ }^{27}$ that for nanotube spectroscopy, the non-symmorphic rod group symmetry of the tubes should be used: As in solid state spectroscopy, where the "point group in the space group" rather than the point group of the unit cell determines the selection rules, it is the "point group in the rod group" that determines which modes are active in infinitely extended nanotubes. In Fig. 1 we demonstrate this for the case of a $(n, n)$ armchair tube. The left hand side shows the unit cell of the tube containing $4 n$ atoms. (Note that the atoms at the lower and upper boundary of the unit cell are also contained in the adjacent unit cells and are therefore counted as "half" atoms.) It can be easily seen that the unit cell has a $n$-fold rotation axis (with rotation angle $\phi=2 \pi / n)$ and a horizontal reflection symmetry plane. Therefore, the point-group symmetry of the unit cell is $C_{n h}$. The right hand side of Fig. 1 demonstrates that in the infinitely extended tube, rotation by $\phi / 2$ with a subsequent translation by the vector $T$ maps the system onto itself as well. The "point group in the rod group" of an armchair BN-tube is therefore the $C_{2 n h}$ group. Table 1 summarizes the findings of Ref. 27 for

Table 1. Symmetries and number of Raman and IR active modes in infinitely long BN nanotubes (following Ref. ${ }^{27}$ )

\begin{tabular}{|l||l|l||l|l||l|l|}
\hline \multicolumn{1}{|c||}{} & \multicolumn{2}{c||}{\begin{tabular}{c} 
armchair $(n, n)$ \\
\multicolumn{1}{|c||}{$C_{2 n h}$}
\end{tabular}} & \multicolumn{2}{c||}{$C_{N}$} & \multicolumn{2}{c|}{$C_{2 n v}$} \\
\hline \hline Raman & $A_{g}$ & 3 & $A$ & 4 & $A_{1}$ & 3 \\
& $E_{1 g}$ & 2 & $E_{1}$ & 5 & $E_{1}$ & 5 \\
& $E_{2 g}$ & 4 & $E_{2}$ & 6 & $E_{2}$ & 6 \\
\hline IR & $A_{u}$ & 1 & $A$ & 4 & $A_{1}$ & 3 \\
& $E_{1 u}$ & 3 & $E_{1}$ & 5 & $E_{1}$ & 5 \\
\hline
\end{tabular}

the IR and Raman active modes in BN-nanotubes. It lists the symmetry groups of armchair, chiral and zigzag $\mathrm{BN}$-nanotubes, the symmetries of the active modes and the corresponding number of modes with that symmetry. 


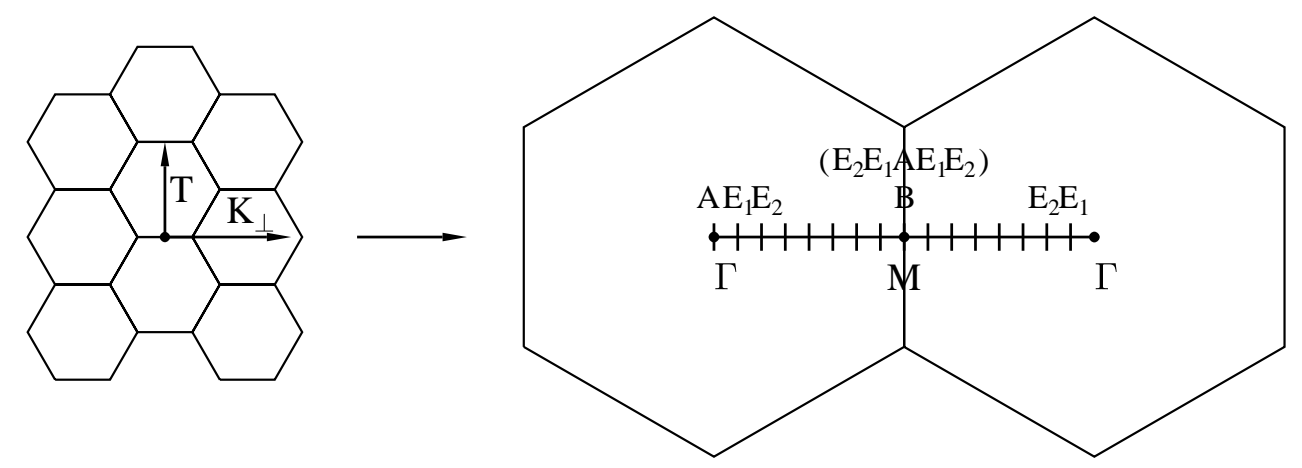

Figure 2. Sketch of the zone-folding method for a $(n, n)$ armchair nanotubes. Left side: hexagonal sheet with translation vector $T$ and perpendicular phonon wave vector $K_{\perp}$ along which the sheet is rolled into a tube. This corresponds to a quantization of $K_{\perp}$ in $2 n$ steps in the Brillouin zone of the sheet along the line $\Gamma \rightarrow \Gamma$ (right hand side of the figure).

Note that for chiral and zigzag tubes, the IR active modes form a subset of the Raman active modes. This is different for armchair tubes where the two sets are disjoint.

The symmetry of the active modes can be understood intuitively by applying the zone-folding method, ${ }^{9}$ i.e. rolling up a sheet into a tube. This is demonstrated for an armchair nanotube in Fig. 2. The left hand side shows a part of a hexagonal sheet which is rolled along the direction of $K_{\perp}$. The phonon wavevector along this direction will be quantized in the tubes. This corresponds to $2 n$ discrete values along the line $\Gamma \rightarrow M \rightarrow \Gamma$ in the reciprocal space of the sheet (right hand side of Fig. 2). The point $\mu=0$ corresponds to an $A$ mode of the tube where all equivalent atoms along the tube circumference move in phase. Fig. 3 a) demonstrates this for the radial buckling mode. (In the high frequency modes, which are derived from the optical phonon branch of the sheet, $\mathrm{B}$ and $\mathrm{N}$ atoms oscillate with a phase difference of $\pi$ while in the low frequency modes, e.g. the radial breathing mode, $\mathrm{B}$ and $\mathrm{N}$ atoms oscillate in phase). The points $\mu=1$ and $\mu=2 n-1$ in Fig. 2 are equivalent and lead to a doubly degenerate $E_{1}$ mode in the tube which has two nodes along the circumference (see Fig. 3 b). Accordingly the two points $\mu=2$ and $\mu=2 n-2$ correspond to a doubly degenerate $E_{2}$ mode with 4 nodes along the circumference (Fig. $3 \mathrm{c}$ ). The construction of the modes $E_{3}, \ldots, E_{n-1}$ works analogously. The point $\mu=n$, finally leads to a non-degenerate $B$ mode where neighboring atoms of one kind oscillate with a phase difference of $\pi$ corresponding to a wave with $n$ nodes along the circumference as depicted in Fig. $3 \mathrm{~d}$ ).
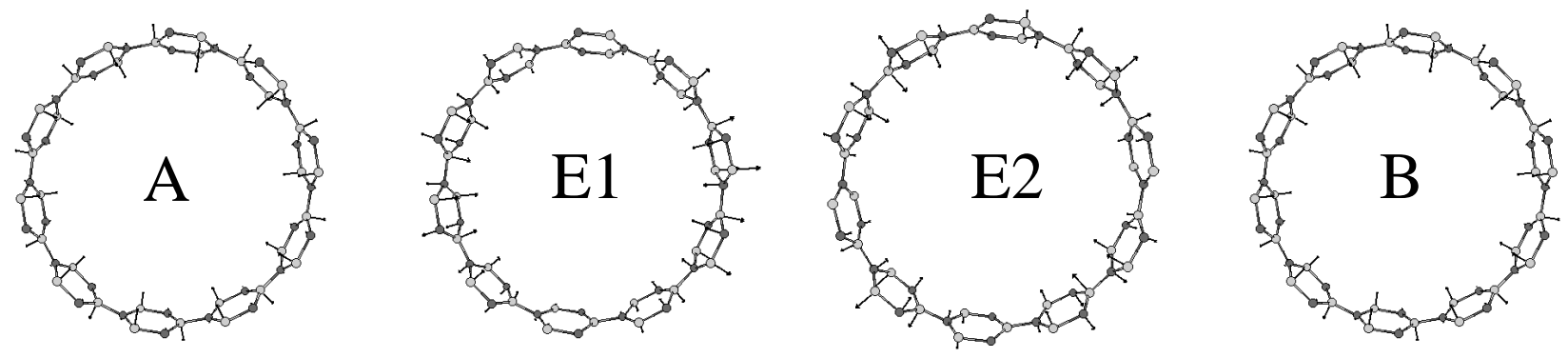

Figure 3. Sketch of the $A, E_{1}, E_{2}$, and $B$ radial buckling modes.

In Fig. 4 we show the $A$ modes of a $(6,6)$ armchair tube that are derived from the three optical modes of the sheet at $\Gamma$. The radial $(\mathrm{R})$ buckling mode stems from the optical out-of plane $(\mathrm{ZO})$ mode of the sheet. The 
two optical in plane modes of the sheet (LO and TO) lead to a mode where the atoms oscillate in transverse or tangential - $(\mathrm{T})$ direction and a mode with oscillation in longitudinal (L) direction. From Fig. 4 it can also be seen that the $\mathrm{R}$ and $\mathrm{T}$ modes have even parity, i.e., they are mapped onto themselves under inversion at the center of the unit cell. The L mode, in contrast, has odd parity because the direction of atomic motion changes under inversion. For chiral and zigzag tubes, there is no inversion symmetry and the distinction between gerade and ungerade modes is absent. It is this presence/absence of inversion symmetry which leads to the above noted fact that the IR active modes are also Raman active in chiral and zigzag tubes while in armchair tubes the two sets have no common elements.
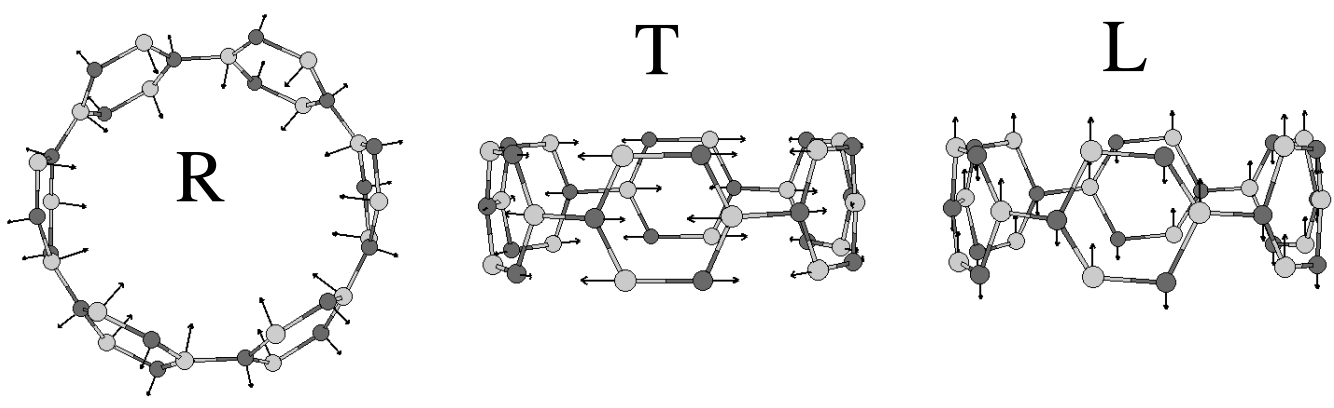

Figure 4. High frequency $A$ modes of a $(6,6)$ armchair nanotube: radial (R), tangential (L), and longitudinal (L) modes.

Fig. 5 displays the $B$ modes of a $(6,6)$ armchair tube which are derived from the $M$ point of the three optical phonon branches of the sheet. As in the case of the $A$ modes, the $\mathrm{R}$ and $\mathrm{T}$ modes have odd parity and the $\mathrm{L}$ mode has even parity. But the behavior under rotation is different from the $A$ modes: The modes are mapped onto themselves under rotation by $\phi=2 \pi / n$ (corresponding to the symmetry group of the unit cell) but they change the direction of atomic motion under the basic symmetry operation of the non-symmorphic rod group which is a rotation by $\phi / 2$ accompanied by translation along the tube-axis.
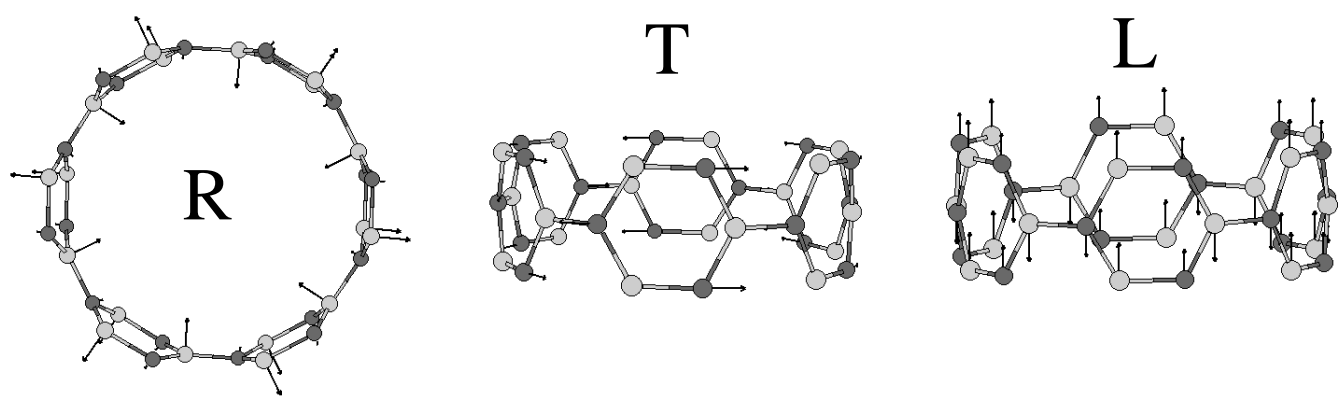

Figure 5. High frequency $B$ modes of a $(6,6)$ armchair nanotube.

The central result of our ab initio calculations is the diameter dependence of the frequencies of Raman and IR active modes which is displayed in Fig. 6 for armchair and zigzag tubes. In the right panel, we also display the phonon dispersion relation of the single sheet in order to illustrate the zone-folding procedure. A detailed discussion of the results can be found in Ref. 26. Here, we just give a summary of the most important features. The phonon spectrum can roughly be divided into three regimes. The phonons in the low frequency region are derived from the acoustic branches of the sheet dispersion relation. With increasing diameter $d$, the phonon frequencies decrease as $1 / d$ or $1 / d^{2}$ corresponding to the liner/quadratic slope of the LA, TA, and ZA branches of the sheet dispersion relation, respectively. It is this strong diameter dependence, which makes the low frequency 
modes, especially the radial breathing mode, prime candidates for the diameter determination via Raman and IR spectroscopy. The phonons in the intermediate frequency region around $800 \mathrm{~cm}^{-1}$ are derived from the optical out of plane $(\mathrm{ZO})$ branch of the sheet. For large diameter, all three phonon modes $\left(A, E_{1}\right.$, and $\left.E_{2}\right)$ converge toward the frequency of the $\mathrm{ZO}$ mode at gamma. This frequency is strongly present in the IR spectra of BN-tubes. ${ }^{24}$ The high frequency regime (above $1200 \mathrm{~cm}^{-1}$ ) comprises the phonon modes derived from the LO and TO branches of the sheet. The L modes converge monotonously increasing toward the asymptotic value at $1382 \mathrm{~cm}^{-1}$ while the $\mathrm{T}$ modes display a strong non-monotonic behavior and approach the asymptotic value from above, corresponding to the strong over-bending of the LO branch in the dispersion relation of the sheet.

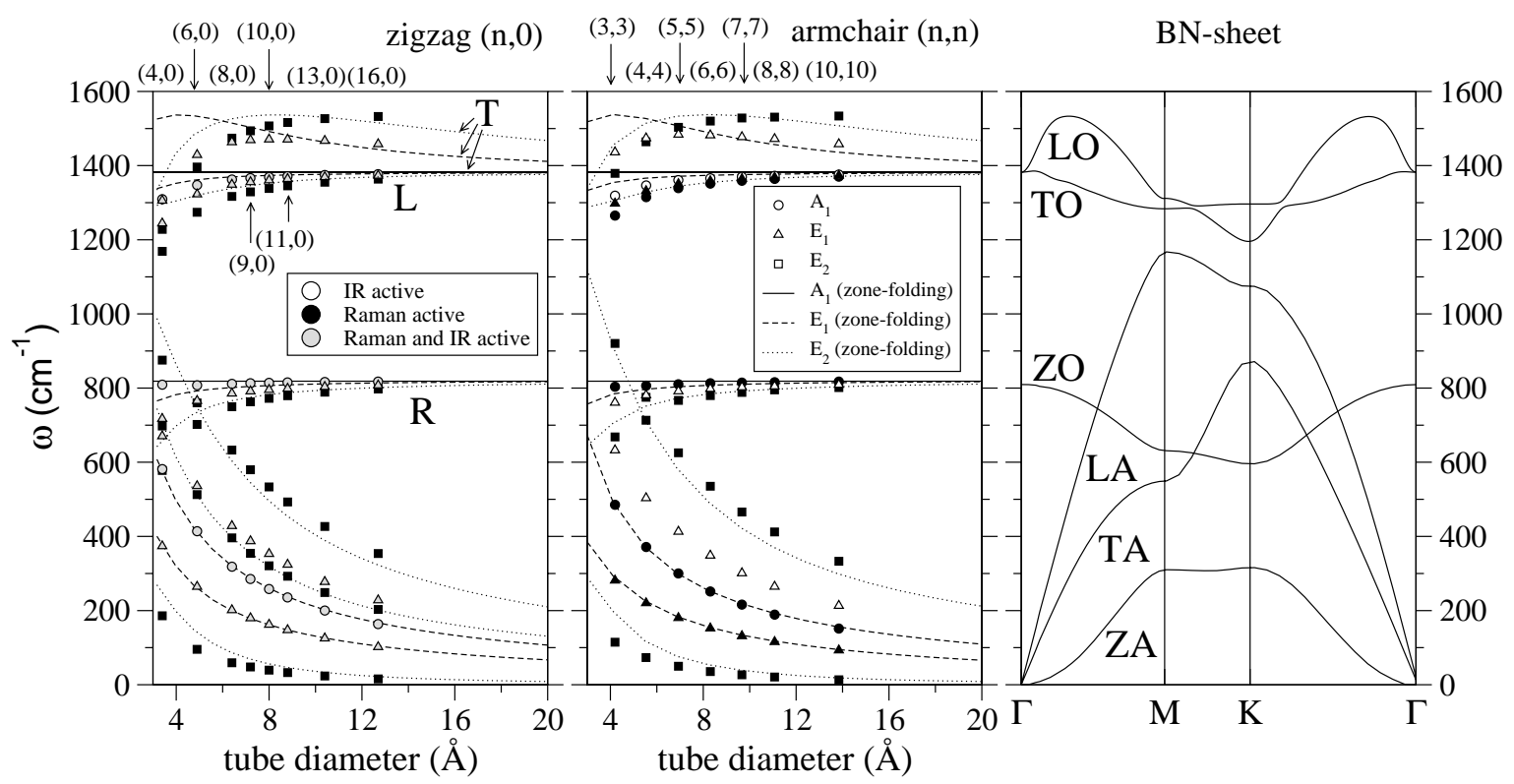

Figure 6. Phonon frequencies of Raman and IR active modes of infinitely long zigzag and armchair BN-nanotubes as a function of the tube diameter. The lines denote the result of the zone-folding method (compare to the dispersion relation of the single sheet in the right panel). The symbols are the result of $a b$ initio calculations for selected tubes. The shape of the symbols characterizes its symmetry (see legend in the inset) and the color distinguishes between modes that are Raman active, IR active or both Raman and IR active.

\section{RAMAN AND IR ACTIVE MODES IN TUBES OF FINITE LENGTHS}

If the tubes are shorter than the wavelength of incident laser light, the tubes appear as long molecules rather than as infinitely extended systems. In this section we discuss the implication of the finite length for the number of Raman and IR active modes. For the symmetry analysis, we assume that the ends of the tubes are terminated in a symmetric way. ${ }^{*}$ The symmetry analysis of chiral and zigzag tubes is unaffected by a non-symmetric termination. However, the destruction of inversion symmetry/ horizontal-reflection symmetry for armchair tubes eliminates the distinction between modes of even and odd parity in that case. For zigzag and chiral nanotubes, the finite length reduces the symmetry of the tube to the point-group symmetry of the unit-cell (assuming that the tube ends do not destroy the point-group symmetry). For $(n, 0)$ zigzag tubes, this means that the symmetry group is $C_{n v}$ and for $(n, m)$ chiral tubes this means that the symmetry group is $C_{d}$, where $d$ is the greatest common divisor between $n$ and $m, \operatorname{gcd}(n, m)$. The case of $(n, n)$ armchair tubes is a little bit more complicated: For an odd number of unit cells in the finite-length tube, the horizontal reflection symmetry of the unit-cell is preserved

\footnotetext{
${ }^{*}$ Note that in BN-systems the formation of $\mathrm{B}-\mathrm{B}$ or N-N bonds is avoided which precludes termination of tubes by fullerene-like half spheres which would contain pentagon rings with two borons or nitrogens as nearest neighbors. ${ }^{40}$ In samples of BN-tubes it was observed that the termination proceeds usually by a flat angular cap or with an encapsulated particle. ${ }^{19}$
} 
and the symmetry group is $C_{n h}$. If the tube contains an even number of unit cells, the reflection symmetry is lost, but the presence of improper rotations enforces the $S_{2 n}$ symmetry of the system. Table 2 summarizes the symmetry groups and the number of active modes for zigzag and armchair tubes of finite-lengths. We have here omitted the case of chiral tubes. If $\operatorname{gcd}(n, m)=1$, in principle all phonon modes can be active. However, the intensities of most of these modes may be vanishingly small. We note that the symmetry evaluation using the point group symmetry of the unit-cell corresponds to the symmetry evaluation which has been mostly employed for the symmetry evaluation of Carbon tubes (up to the very recent accurate $a b$ initio calculations in Ref. 30). In the zone-folding picture, the use of the point-group symmetry of the unit cell signifies that not only the modes of the $\Gamma$-point of the sheet, but also the modes of the $M$-point are mapped onto $A$ modes of the tube. This is indicated in Fig. 2 by the letters in parentheses. Furthermore, the modes close to the $M$-point which would fold onto modes of $E_{n-1}$ and $E_{n-2}$ symmetry according to the strict symmetry assignment, posses $E_{1}$ and $E_{2}$ symmetry according to the more relaxed point-group symmetry of the unit-cell.

Table 2. Symmetries and number of Raman and IR active modes in finite chiral and armchair BN nanotubes.

\begin{tabular}{|c|c|c|c|c|c|c|c|c|c|c|}
\hline & \multicolumn{8}{|c|}{ armchair $(n, n)$} & \multirow{3}{*}{\multicolumn{2}{|c|}{$\begin{array}{c}\operatorname{zigzag}(n, 0) \\
C_{n v}\end{array}$}} \\
\hline & \multicolumn{4}{|c|}{$\begin{array}{c}C_{n h} \\
\text { (even number of unit cells) }\end{array}$} & \multicolumn{4}{|c|}{$\begin{array}{c}S_{2 n} \\
\text { (odd number of unit cells) }\end{array}$} & & \\
\hline & \multicolumn{2}{|c|}{$n$ even } & \multicolumn{2}{|c|}{$n$ odd } & \multicolumn{2}{|c|}{$n$ even } & \multicolumn{2}{|c|}{$n$ odd } & & \\
\hline \multirow[t]{3}{*}{ Raman } & $A_{g}$ & $\overline{77}$ & $A^{\prime}$ & 7 & $A$ & 4 & $A_{g}$ & 4 & $A_{1}$ & 7 \\
\hline & $E_{1 g}$ & 4 & $E 1^{\prime \prime}$ & 4 & $E_{1}$ & 11 & $E_{1 g}$ & 4 & $E_{1}$ & 11 \\
\hline & $E_{2 g}$ & 8 & $E 2^{\prime}$ & 8 & $E_{2}$ & 12 & $E_{2 g}$ & 8 & $E_{2}$ & 12 \\
\hline \multirow[t]{2}{*}{ IR } & $A_{u}$ & 3 & $A^{\prime \prime}$ & 3 & $B$ & 4 & $A_{u}$ & 4 & $A_{1}$ & 7 \\
\hline & $E_{1 u}$ & 7 & $E 1^{\prime}$ & 7 & $E_{1}$ & 11 & $E_{1 u}$ & 7 & $E_{1}$ & 11 \\
\hline
\end{tabular}

The diameter dependence of all active modes in finite-length tubes is displayed in Fig. 7. In comparison with Fig. 6, additional branches appear which converge toward the phonons of the sheet at the $M$-point with frequencies $305 \mathrm{~cm}^{-1}, 550 \mathrm{~cm}^{-1}, 636 \mathrm{~cm}^{-1}, 1168 \mathrm{~cm}^{-1}, 1283 \mathrm{~cm}^{-1}$, and $1315 \mathrm{~cm}^{-1}$. Note the pronounced difference in the scaling with diameter of the additional branches between armchair and zigzag tubes. E.g., the branch that approaches $1168 \mathrm{~cm}^{-1}$ is very flat in the case of the zigzag tubes but has a strong diameter dependence for the armchair tubes. This is easily understood when keeping in mind that for zigzag tubes, the zone-folding is done along the line $\Gamma \rightarrow K \rightarrow M \rightarrow K \rightarrow \Gamma$ in the Brillouin zone of the sheet. This means that in the dispersion relation of the sheet (see right panel of Fig. 6), the $M$-point at $1168 \mathrm{~cm}^{-1}$ is approached from the right side where the dispersion relation is very flat. For armchair tubes, the $M$-point is accordingly approached from the left side where the dispersion relation is steep. In the case of the phonon branch approaching $550 \mathrm{~cm}^{-1}$ for $d \rightarrow \infty$, this effect is even stronger and leads to a monotonously decreasing convergence toward this value for zigzag tubes and to a monotonously increasing convergence for armchair tubes.

For the proposed frequencies of active modes in tubes of finite length in Fig. 7 we have assumed that the tubes are sufficiently long such that their frequencies closely resemble the frequencies of the infinitely long tube. In other words, we have used the phonons modes at $\Gamma$ of the infinitely long tube and performed the symmetry analysis as if the tubes had finite length. This approximation is justified for vibrational modes with very long wave-length along the tube axis. Modes with short wave-length along the tube axis have considerably different frequencies which resemble the frequencies of phonons with non-vanishing $q$ in infinite tubes. However, the Raman and IR intensities are vanishingly small, because the strong oscillations lead to very weak couplingmatrix elements with the laser field. Furthermore, the fact that a mode is "allowed" by the selection rules does not mean that it has a high intensity. The intensity of the modes that are allowed in finite tubes but forbidden in infinitely long tubes decays with the length of the tube. If the exact scaling is calculated, the intensities of these peaks can be used to determine the tube length. 


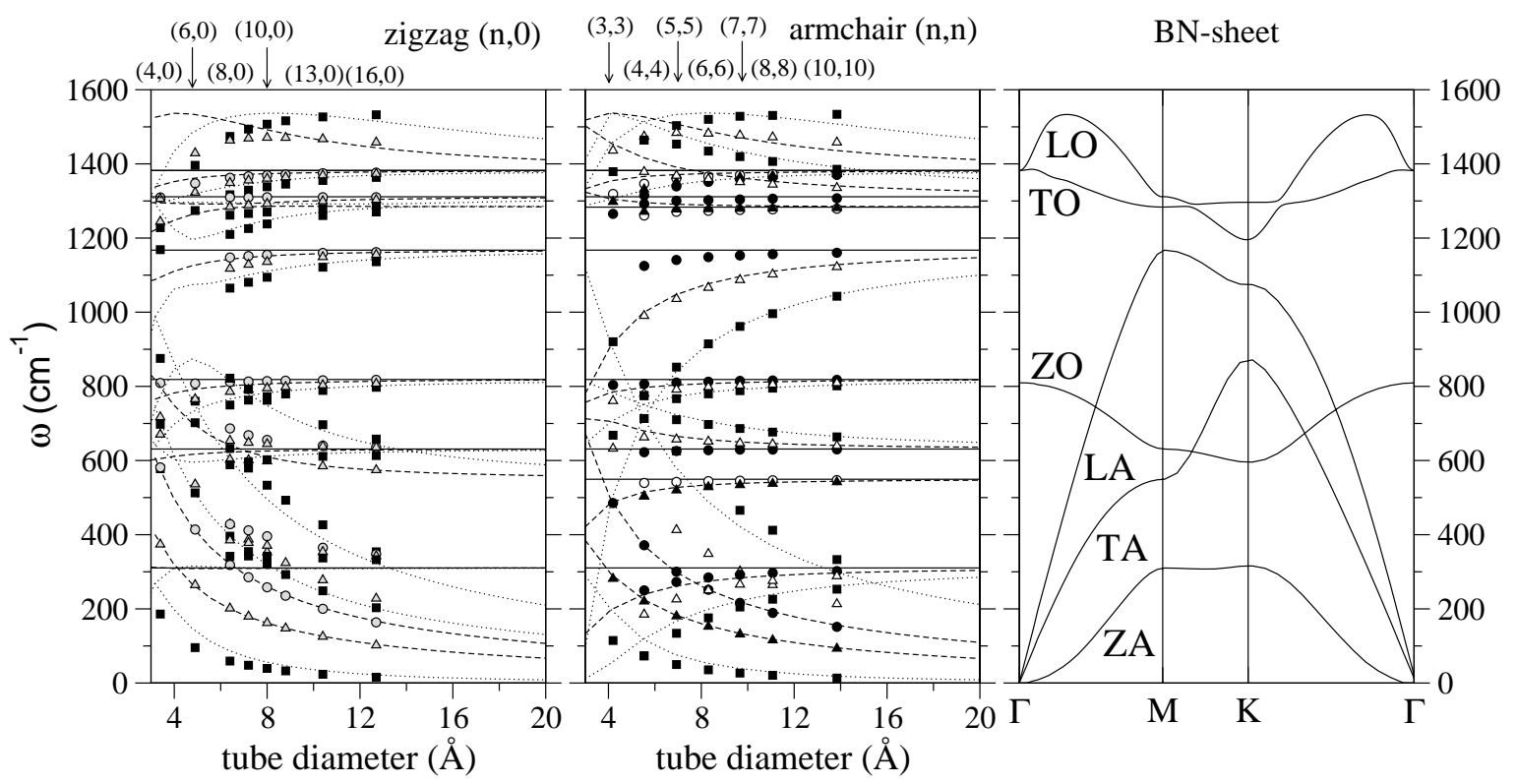

Figure 7. Raman and IR active modes of BN-nanotubes with finite lengths. For an explanation of the symbols see Fig. 6.

\section{PHONON FREQUENCIES IN A DENSELY PACKED PERIODIC ARRAY OF TUBES}

Since in real samples ${ }^{19,24}$ nanotubes occur both isolated and in bundles, it is important to investigate the effect of inter-tube interaction onto the phonon frequencies. For carbon nanotubes, only a weak perturbation was found for closely packed nanotube arrays. In the calculations of Kahn et al. ${ }^{41}$ the effect of bundling does not exceed $10 \mathrm{~cm}^{-1}$ except for the very low energy $E_{1}$ and $E_{2}$ modes. For the RBM, they found a stiffening by $4 \%$. Henrard et al. ${ }^{42}$ found a value of $10 \%$ for the stiffening of the RBM due do bundling.

We investigate the effect of bundling in BN-nanotubes by calculating a densely packed lattice of nanotubes with an inter-tube distance of $6.5 \mathrm{a}$.u. which corresponds approximately to the inter-sheet distance in h-BN. As in the case of "isolated" tubes, the geometry is optimized which leads to a slight deformation from the ideal, cylindrical geometry and requires the displacement of all atoms in the unit cell for the calculation of phonon frequencies. In the upper part of Table 3, we compare the (Raman and IR active) phonon frequencies of a close-packed $(8,0)$ tube with a (quasi-)isolated $(8,0)$ tube. The general effect of bundling is a slight shift of the non-degenerate $A$ modes and a splitting of the doubly degenerate $E$ modes into two modes with slightly different frequencies. Most phonon modes (except for the lowest $E_{2 g}$ mode) are softened, contrary to the observations in $\mathrm{C}$ nanotubes where - on average - a slight stiffening of the modes was observed. ${ }^{41,42}$ Apparently, the electrostatic inter-tube interaction acts, on average, as an attractive force which counteracts the hardening of phonons that would take place if only the repulsion caused by the beginning inter-tube overlap of the wave-functions would modify the frequency. The effect, however, is weak and does not exceed $10 \mathrm{~cm}^{-1}$ in most cases. A notable exception to the weak influence of bundling is the high frequency tangential $E_{1}(\mathrm{~T})$ mode, which is softened by almost $50 \mathrm{~cm}^{-1}$. This mode is depicted in Fig. 8. At the distance of closest approach between neighboring tubes, equivalent atoms are locally moving like in two parallel planes. This softens the mode and makes it susceptible to LO-TO splitting in macroscopically extended nanotube-arrays as shown below. In C-nanotubes, where the electrostatic interaction is absent, this $E_{1}$ modes do not experience a different shift by bundling than the $A$ and $E_{2}$ modes.

In macroscopic polar crystals, the longitudinal optical phonons give rise to an electric field which leads to a shift in frequency and to a splitting of degenerate LO/TO modes. For bulk h-BN, this LO-TO splitting amounts to almost $200 \mathrm{~cm}^{-1}$ (Ref. 6). "Macroscopic" in this context means that the dimensions of the "crystal" in all three directions are larger than the wave-length of the interacting laser light. While this is certainly not the 
Table 3. Change of phonon frequencies (in $\mathrm{cm}^{-1}$ due to close packing of tubes in a periodic array: Raman and IR active modes for an isolated $\mathrm{BN}(8,0)$ tube (with an intertube distance of 14 a.u.) in comparison with the modes for a "close packed" array of tubes (intertube distance 7 a.u.). If the coupling to an electric field is included, a strong shift/splitting of some frequencies is observed. (The numbers in parantheses are the corresponding frequencies without E-field coupling).

\begin{tabular}{|c|c|c|c|}
\hline \multicolumn{4}{|c|}{ Without coupling to macroscopic E-field } \\
\hline & & isolated $\mathrm{BN}(8,0)$ & close packed $\mathrm{BN}(8,0)$ \\
\hline$E_{2}$ & $\mathrm{R}$ & 59.3 & $66.5 / 76.5$ \\
\hline$E_{1}$ & $\mathrm{~L}$ & 201.4 & $200.2 / 201.0$ \\
\hline$A_{1}$ & $\mathrm{R}$ & 317.9 & 318.1 \\
\hline$E_{2}$ & $\mathrm{~L}$ & 396.0 & $395.6 / 396.0$ \\
\hline$E_{1}$ & $\mathrm{~T}$ & 428.4 & $422.3 / 431.0$ \\
\hline$E_{2}$ & $\mathrm{~T}$ & 632.8 & $630.3 / 632.6$ \\
\hline$E_{2}$ & $\mathrm{R}$ & 749.8 & $746.8 / 747.2$ \\
\hline$E_{1}$ & $\mathrm{R}$ & 785.5 & $773.9 / 775.5$ \\
\hline$A_{1}$ & $\mathrm{R}$ & 808.5 & 805.7 \\
\hline$E_{1}$ & $\mathrm{~L}$ & 1348.9 & $1346.8 / 1347.1$ \\
\hline$A_{1}$ & $\mathrm{~L}$ & 1362.5 & 1361.4 \\
\hline$E_{1}$ & $\mathrm{~T}$ & 1463.4 & 1416.6/1417.8 \\
\hline$E_{2}$ & $\mathrm{~T}$ & 1474.0 & $1465.1 / 1470.4$ \\
\hline \multicolumn{4}{|c|}{ E-field coupling with $q \rightarrow 0$ along tube axis } \\
\hline & & isolated $\mathrm{BN}(8,0)$ & close packed $\mathrm{BN}(8,0)$ \\
\hline$A_{1}$ & $\mathrm{R}$ & $318.5(317.9)$ & $318.7(318.1)$ \\
\hline$A_{1}$ & $\mathrm{R}$ & $811.6(808.5)$ & $809.0(805.6)$ \\
\hline$A_{1}$ & $\mathrm{~L}$ & $1547.2(1362.5)$ & $1585.9(1361.5)$ \\
\hline \multicolumn{4}{|c|}{ E-field coupling with $q \rightarrow 0$ perpendicular to tube axis } \\
\hline & & isolated $\mathrm{BN}(8,0)$ & close packed $\mathrm{BN}(8,0)$ \\
\hline$E_{1}$ & $\mathrm{~T}$ & $428.4 / 428.7(428.4)$ & $422.4 / 431.5(422.3 / 431.0)$ \\
\hline$E_{1}$ & $\mathrm{R}$ & $785.7 / 791.9(785.5)$ & $775.0 / 791.4(773.9 / 775.5)$ \\
\hline$E_{1}$ & $\mathrm{~L}$ & 1348.9/1349.0 (1348.9) & $1346.9 / 1347.2(1346.8 / 1347.1)$ \\
\hline$E_{1}$ & $\mathrm{~T}$ & $1463.4 / 1500.8(1463.4)$ & $1416.9 / 1507.3(1416.6 / 1417.8)$ \\
\hline
\end{tabular}

case for nanotube bundles containing only tens of nanotubes, it is conceivable that in the near future, better purification and alignment methods lead to large periodic arrays of nanotubes. In this case, the effect of coupling to the E-field will become important for the longitudinal optical (LO) modes. In the following, LO means always longitudinal with respect to the wave-vector of the interacting laser light. Even though the excited phonons close to $\Gamma$ carry approximately zero-momentum, it does play a role from which direction the limit $\vec{q} \rightarrow 0$ is taken. Table 3 demonstrates that for the laser light parallel to the tube axis, the $A_{1}$ modes experience a shift. In the case of the radial breathing and buckling modes, the shift is weak. However for the high-frequency $A_{1}(\mathrm{~L})$ mode, the shift even exceeds the $200 \mathrm{~cm}^{-1}$ of the LO-TO splitting in h-BN. Note that the shift is also present for the periodic array of tubes with large inter-tube distance. Due to the long-range electro-static interactions between the tubes, the shift decreases very slowly as a function of inter-tube distance. For incidence of the laser light perpendicular to the tube axis, it is the $E_{1}$ modes which experience a splitting in frequency. While all $E_{1}$ modes are slightly affected, it is the $E_{1}(\mathrm{~T})$ mode of Fig. 8 which experiences the relatively strong splitting of about $90 \mathrm{~cm}^{-1}$ in the close packed array and $40 \mathrm{~cm}^{-1}$ in the array with large inter-tube distance. It can be seen from Fig. 8 that all $\mathrm{B}$ atoms are moving parallel and the $\mathrm{N}$ atoms likewise. This makes it a longitudinal optical mode in the macroscopic array of tubes with the laser light incident in the direction of oscillation. In anisotropic polar systems like h-BN, the directional dependence of the LO-TO splitting has been used for angular resolved spectroscopy (ellipsometry). ${ }^{4}$ Due to the variety of modes, macroscopic arrays of BN-nanotubes promise to be an even richer playground for angular resolved IR spectroscopy. 


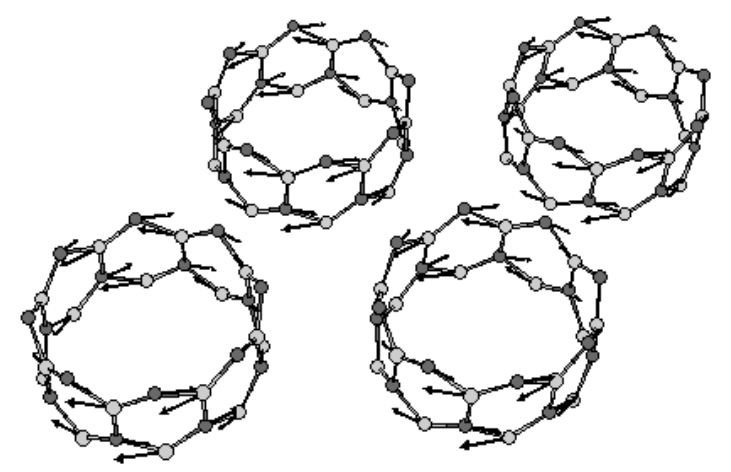

Figure 8. High frequency tangential $E_{1}$ mode in a close packed array of $(8,0)$ BN-nanotubes.

\section{CONCLUSION}

Since BN-nanotubes can be now produced in macroscopic (i.e., gram) quantities, Raman and IR spectroscopy will very likely develop into standard tools for characterization of the tubes according to diameter, length, and chirality. The characterization requires accurate data on the vibrational properties of the tubes. We have presented $a b$ initio calculations of the phonon frequencies as a function of tube diameter and tube chirality. The phonon modes display a very close similarity to the phonon modes in carbon tubes. However, due to the reduced rod group symmetry more modes are Raman and IR active. We have discussed possible deviations from the phonon frequencies of ideal, isolated and infinitely long tubes in realistic samples of BN-tubes. The finite length of the tube (typically shorter than the wave length of infrared light) leads to a reduction of the tube symmetry and thereby to additional active modes. The Raman and IR intensity of these modes might serve as an indicator for the average length of the measured tubes. An important difference with respect to carbon tubes is the polarity of BN. In large arrays of densely packed tubes, the polarity leads to a coupling of longitudinal optical modes with the internal electric field. The corresponding frequency shift of these modes depends on the direction of the incoming light and the average distance between the tubes. The richness of predicted phonon frequencies gives rise to the hope, that in the future large samples of clean and well-aligned samples can be produced and spectroscopically investigated.

\section{ACKNOWLEDGMENTS}

We acknowledge stimulating discussions with Francesco Mauri, Michele Lazzeri, Ofir Alon, Raul Arenal de la Concha, and Annick Loiseau. Work supported by COMELCAN (HPRN-CT-2000-00128), MCyT (Grant: MAT2001-0946), and the University of the Basque Country (Contract 00206.215-13639/2001). Part of the calculations was performed at CEPBA (Barcelona).

\section{REFERENCES}

1. R. Geick, C. H. Perry, and G. Rupprecht, Phys. Rev. 146, 543 (1966).

2. D. M. Hoffmann, G. L. Doll, and P. C. Eklund, Phys. Rev. B 30, 6051 (1984).

3. X. Blase, A. Rubio, S. G. Louie, and M. L. Cohen, Phys. Rev. B 51, 6868 (1995).

4. M. Schubert, B. Rheinländer, E. Franke, H. Neumann, T. E. Tiwald, J. A. Woollam, J. Hahn, and F. Richter, Phys. Rev. B 56, 13306 (1997).

5. E. Rokuta, Y. Hasagawa, K. Suzuki, Y. Gamou, C. Oshima, and A. Nashima, Phys. Rev. Lett. 79, 4609 (1997).

6. G. Kern, G. Kresse, and J. Hafner, Phys. Rev. B 59, 5881 (1999).

7. A. Rubio, J. L. Corkill, and M. L. Cohen, Phys. Rev. B 49, 5081 (1994).

8. X. Blase, A. Rubio, S. G. Louie, and M. L. Cohen, Europhys. Lett 28, 335 (1994).

Proc. of SPIE Vol. 5118 
9. R. Saito, G. Dresselhaus, and M. S. Dresselhaus, Physical Properties of Carbon Nanotubes (Imperial College Press, London, 1998).

10. R. A. Jishi, L. Venkataraman, M. S. Dresselhaus, and G. Dresselhaus, Chem. Phys. Lett. 209, 77-82 (1993).

11. N. G. Chopra, J. Luyken, K. Cherry, V. H. Crespi, M. L. Cohen, S. G. Louie, and A. Zettl, Science 269, 966 (1995).

12. A. Loiseau, F. Willaime, N. Demoncy, G. Hung, and H. Pascard, Phys. Rev. Lett. 76, 4737 (1996).

13. D. P. Yu, X. S. Sun, C. S. Lee, I. Bello, S. T. Lee, H. D. Gu, K. M. Leung, G. W. Zhou, Z. F. Dong, and Z. Zhang, Appl. Phys. Lett. 72, 1966 (1998).

14. D. Goldberg, Y. Bando, W. Han, K. Kurashima, and T. Sato, Chem. Phys. Lett. bf 308, 337 (1999).

15. Y. Chen, J. F. Gerald, J. S. Williams, and S. Bulcock, Chem. Phys. Lett. 299, 260 (1999).

16. J. Cummings and A. Zettl, Chem. Phys. Lett 316, 211 (2000).

17. T. Laude, Y. Matsui, A. Marraud, and B. Jouffrey, Appl. Phys. Lett. 76, 3239 (2000).

18. E. Bengu and L. D. Marks, Phys. Rev. Lett 86, 2385 (2001).

19. R. S. Lee, J. Gavillet, M. Lamy de la Chapelle, A. Loiseau, J.-L. Cochon, D. Pigache, J. Thibault, and F. Willaime, Phys. Rev. B 64, 121405(R) (2001).

20. L. C. Venema, J. W. Janssen, M. R. Buitelaar, J. W. G. Wildöer, S. G. Lemay, L. P. Kouwenhoven, and C. Dekker, Phys. Rev. B 62, 5238 (2000).

21. S. M. Bachilo, M. S. Strano, C. Kittrell, R. H. Hauge, R. E. Smalley, and R. B. Weisman, Sciene 298, 261 (2003).

22. A. M. Rao, E. Richter, S. Bandow, B. Chase, P. C. Eklund, K. A. Williams, S. Fang, K. R. Subbaswamy, M. Menon, A. Thess, R. E. Smalley, G. Dresselhaus, and M. S. Dresselhaus, Science 275, 187 (1997).

23. R. Saito, T. Takeya, T. Kimura, G. Dresselhaus, and M. S. Dresselhaus, Phys. Rev. B 57, 4145 (1998).

24. R. Arenal de la Concha, L. Wirtz, J.-Y. Mevellec, S. Lefran, A. Loiseau, and A. Rubio, submitted (2002).

25. U. Kuhlmann, H. Jontoljak, N. Pfänder, P. Bernier, C. Journet, and C. Thomsen, Chem. Phys. Lett. 294, 237 (1998).

26. L. Wirtz, Angel Rubio, R. Arenal de la Concha, and A. Loiseau, submitted to PRB (2003).

27. O. E. Alon, Phys. Rev. B 64, 153408 (2001).

28. M. Damnjanović, T. Vuković, I. Milošević, and B. Nikolić, Act Cryst. A57, 304 (2001).

29. N. W. Ashcroft and N. D. Mermin, Solid State Physics, Saunders College Publishing, Orlando, 1976.

30. O. Dubay and G. Kresse, Phys. Rev. B 67, 035401 (2003).

31. J. Yu, R. K. Kalia, and P. Vashishta, J. Chem. Phys. 103, 6697 (1995)

32. D. Sánchez-Portal, E. Artacho, J. M. Soler, A. Rubio, and P. Ordejón, Phys. Rev. B 59, 12678 (1999).

33. D. Sánchez-Portal and E. Hernández, Phys. Rev. B 66, 235415 (2002).

34. V. N. Popov, Phys. Rev. B 67, 085408 (2003).

35. The ABINIT code is a common project of the Université Catholique de Louvain, Corning Incorporated, and other contributors (URL http://www.abinit.org).

36. N. Troullier and J. L. Martins, Phys. Rev. B 43, 1993 (1991).

37. S. Baroni, S. de Gironcoli, A. Dal Corso, and P. Giannozzi, Rev. Mod. Phys. 73, 515 (2001).

38. X. Gonze, Phys. Rev. B 55, 10337 (1997); X. Gonze and C. Lee, Phys. Rev. B 55, 10355 (1997).

39. W. Kohn and L. J. Sham, Phys. Rev. 140, A1133 (1965).

40. G. Seifert, P. W. Fowler, D. Mitchell, D. Porezag, and Th. Frauenheim, Chem. Phys. Lett. 268, 352 (1997).

41. D. Kahn and J. P. Lu, Phys. Rev. B 60, 6535 (1999).

42. L. Henrard, E. Hernández, P. Bernier, and A. Rubio, Phys. Rev. B 60, 8521 (1999). 